\title{
NON-EQUIDISTANT "BASIC FORM"-FOCUSED GREY VERHULST MODELS (NBFGVMS) FOR ILL-STRUCTURED SOCIO-ECONOMIC FORECASTING PROBLEMS
}

\author{
Mohammad HASHEM-NAZARI ${ }^{1}$, Akbar ESFAHANIPOUR ${ }^{2}$, \\ S.M.T. FATEMI GHOMI ${ }^{3}$
}

Department of Industrial Engineering and Management Systems, Amirkabir University of Technology, 424 Hafez Ave, 15875-4413, Tehran, Iran E-mails: ${ }^{2}$ hashem.nazari@aut.ac.ir; ${ }^{2}$ esfahaa@aut.ac.ir (correspondingauthor); 3atemi@aut.ac.ir

Received 23 February 2017; accepted 29 May 2017

\begin{abstract}
Multiple uncertainties complicate socio-economic forecasting problems, especially when relying on ill-conditioned limited data. Such problems are best addressed by grey prediction models such as Grey Verhulst Model (GVM). This paper resolves the incompatibility between GVM's estimation and prediction by taking its basic form equation as the basis of both. The resultant "Basic Form"-focused GVM (BFGVM) is also further developed to create Direct Non-equidistant BFGVM (DNBFGVM) and, in turn, DNBFGVM with Recursive simulation (DNBFGVMR). Experimental analyses comprise 19 socio-economic time series with an emphasis on Iranian population, a low-frequency non-equidistant time series with remarkable strategic importance. Promisingly, the proposed DNBFGVM and DNBFGVMR provide accurate in-sample and out-of-sample socio-economic forecasts, show highly significant improvements over the best traditional GVM, and offer cost-effective intelligent support of decision-making. Final results suggest future trends of studied socio-economic time series. Specifically, they reveal Iranian population to grow even slower than anticipated, demanding an urgent consideration of policy-makers.
\end{abstract}

Keywords: grey system theory, direct grey Verhulst model, discretisation, limited data, population forecasting, nonparametric statistical tests.

JEL Classification: C14, C22, E27, J11.

Online supplementary material: Supporting information for this paper is available as online supplementary material at https://doi.org/10.3846/16111699.2017.1337045

\section{Introduction}

Conventional techniques have become increasingly ineffective in forecasting complex socio-economic phenomena (Esfahanipour et al. 2016). Additionally, socio-economic researches in less-developed countries often rely on insufficient observations, i.e. lowfrequency time series as well as irregular data collection, i.e. non-equidistant time series. Such ill-structured forecasting problems are best addressed by grey prediction. 
A major drawback in grey prediction is inherent incompatibility between continuous prediction (simulation) and discrete estimation. This research gap has been narrowed e.g. Wang, Z.-X. et al. (2007), Xiao and Qin, L.-F. (2012), and Xie and Liu (2009), but it persists particularly for GVM.

This paper resolves incompatibility between GVM's prediction and estimation. It builds on basic form equation to equip GVM with cohesive discrete forecasting procedure and to establish "Basic Form"-focused GVM (BFGVM). Considering irregularities prevalent in socio-economic time series, we create Non-equidistant BFGVM (NBFGVM). Then, it is promoted to Direct NBFGVM (DNBFGVM) adaptable to strictly increasing data. Revising forecasting functions to form recursive simulation, we then develop NBFGVMR and DNBFGVMR.

19 time series in various socio-economic fields are employed to validate proposed models. Meanwhile, having roots in Verhulst population model, they can best be validated through population time series. The case of the Islamic Republic of Iran is striking because of its unique demographic trends (Mahmoodi et al. 2015). After years of fastpaced social change (e.g. Islamic revolution, Iraqi invasion, and subsequent 8 years' war) and resultant fluctuating demographic behaviour, it has started a long-lasting and steady family planning programme. The programme met its official objectives several years ago (Vahidnia 2007); albeit, it persists. Preserving the status quo may give rise to unintended consequences such as labour shortage (Erfani 2013; Jafari et al. 2017) and ageing (Jafari et al. 2017). Reconsidering the strict antinatalist policy (Erfani 2013; Jafari et al. 2017; Mahmoodi et al. 2015), authorities require population forecasts as necessary decision support. Applying grey models is nearly inevitable since censuses provide a sequence limited only to seven non-equidistant points.

Proposed models are expected to make remarkable improvements in socio-economic forecasting. They are especially promising where sufficiently knowledgeable experts are inaccessible or too costly. Indeed, these models can even provide experts with decision support.

The paper is organised as follows. Section 1 reviews the literature of grey prediction and emphasises research gaps. Section 2 explains research data followed by computational procedure of every (traditional or proposed) model. Section 3 provides performance evaluation framework followed by practical comparative analyses in forecasting socioeconomic time series in general and the population in particular. Section 4 integrates socio-economic and population results to recommend specific models and then modifies their training to derive applicable extrapolations. Lastly, conclusions are drawn and guidelines are issued for future researchers.

\section{Literature review}

Deng named his theory "grey" (blackish white) because it is particularly aimed at systems with partially unknown features (Kayacan et al. 2010; Liu, Lin 2010; Tsai et al. 2017). Grey prediction is a main component of grey system theory having plentiful advantages such as easily-interpretable structure (Bezuglov, Comert 2016), simple com- 
putation (Hu, Qin, Y.-L. 2011; Li et al. 2011), adaptability to ill-conditioned data (Hu, Qin, Y.-L. 2011; Li et al. 2011; Bezuglov, Comert 2016), minimal assumptions about distributions of samples (Tsai et al. 2017), requiring as few as four pieces of data ( $\mathrm{Li}$ et al. 2011; Tsai et al. 2017; Bezuglov, Comert 2016), and superior performance under poor information (Wang, X.-Q. et al. 2014; Zhou, He, J.-M. 2013). Thus, it has growing popularity among forecasting researchers (Kayacan et al. 2010).

First-order grey models are basic techniques, sacrificing flexibility for simplicity $(\mathrm{Hu}$, Qin, Y.-L. 2011). More flexible higher-order models, on the other hand, are hardly employed mostly because of their complexity (Kayacan, Kaynak 2011). Grey system theorists have dealt with this dilemma by developing nonstandard models incorporating nonlinear features into first-order models.

Among such models, Grey Verhulst Model (GVM) is especially popular as Liu and Lin (2010), Wang, Z.-X. et al. (2009), and Xiao and Qin, L.-F. (2012) emphasise its widespread applications, Wang, Y. et al. (2009) suggest its frequent use as a typical grey model, and Xiao and Qin, L.-F. (2012) declare its importance as a nonlinear grey model. It is a current area of abundant research as Table 1 outlines.

There is well-recognised incompatibility between prediction (simulation) and estimation of grey prediction models, as mentioned in the Introduction. As far as we know,

Table 1. Major research trends on grey Verhulst model compared with this research

\begin{tabular}{|c|c|c|c|c|c|c|c|c|c|c|c|}
\hline & 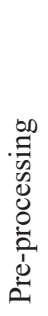 & 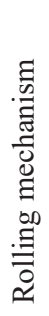 & 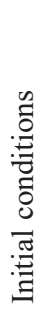 & 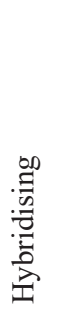 & 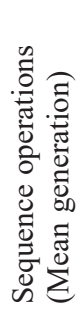 & 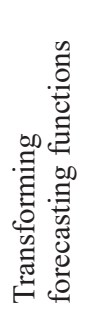 & 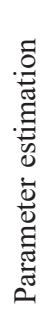 & 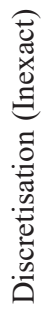 & 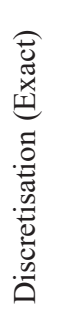 & 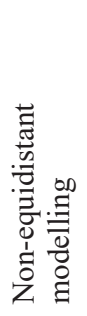 & 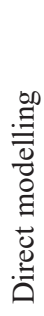 \\
\hline Wang, Z.-X. et al. (2007) & & & & & $\checkmark$ & & $\checkmark$ & & & & $\checkmark$ \\
\hline Luo and He, Z.-M. (2009) & & & $\checkmark$ & & & & & & & $\checkmark$ & $\checkmark$ \\
\hline Wang, Y. et al. (2009) & $\checkmark$ & $\checkmark$ & & $\checkmark$ & & & & & & & $\checkmark$ \\
\hline Wang, Z.-X. et al. (2009) & $\checkmark$ & & $\checkmark$ & & & & & $\checkmark$ & & & \\
\hline Kayacan et al. (2010) & $\checkmark$ & $\checkmark$ & & $\checkmark$ & & & & & & & \\
\hline Xiong et al. (2010) & & & $\checkmark$ & & & & & & & $\checkmark$ & \\
\hline Shu et al. (2011) & $\checkmark$ & & & & & & & & & $\checkmark$ & $\checkmark$ \\
\hline Xiao and Qin, L.-F. (2012) & $\checkmark$ & & & & & & & $\checkmark$ & & & \\
\hline Guo et al. (2013) & & $\checkmark$ & & $\checkmark$ & $\checkmark$ & $\checkmark$ & & $\checkmark$ & & & \\
\hline Evans (2014) & & $\checkmark$ & & & & $\checkmark$ & $\checkmark$ & & & & \\
\hline Wang, X.-Q. et al. (2014) & & & & & & & & & & & $\checkmark$ \\
\hline Bezuglov and Comert (2016) & & $\checkmark$ & & $\checkmark$ & & & & & & & \\
\hline This research & & & & & & & & & $\checkmark$ & $\checkmark$ & $\checkmark$ \\
\hline
\end{tabular}


Z.-X. Wang and his colleagues are the first to focus on such a drawback in GVM (Wang, Z.-X. et al. 2007; Wang, Z.-X. et al. 2009). They diagnose an unjustifiable span between GVM's difference-equation-based parameter estimation and its differentialequation-based forecasting functions. Consequently, estimated parameters intrinsically fail to match forecasting functions. Xiao and Qin, L.-F. (2012) are in perfect agreement in that they introduce the same sudden transition between different equations as the cause of large errors in GVM. Furthermore, this drawback has also been identified as the main source of errors in first-order (Liu, Lin 2010; Xie, Liu 2009; Zhou, He, J.-M. 2013) and second-order (Li et al. 2011) models.

Xie and Liu (2009) developed Discrete Grey Model to avoid such incompatibility. Unfortunately, their technique is hardly applicable to GVM, though some scholars have implemented it in an approximate manner (Wang, Z.-X. et al. 2009; Xiao, Qin, L.-F. 2012). To the best of our knowledge, Guo et al. (2013) are the only researchers who discretise GVM without relying on Discrete Grey Model. Nonetheless, they do not compensate the lack of an exact discretisation of GVM since they concentrate on a completely transformed GVM.

Besides, the classical assumption of regular temporal structure in data is often violated in the real-world. Accordingly, scholars have generalised GVM to handle non-equidistant data (Luo, He, Z.-M. 2009; Shu et al. 2011; Xiong et al. 2010).

In addition, not every saturating trend reaches a peak and then declines. In fact, numerous saturating trends continuously approach a limit and never touch it. Accordingly, researchers have implemented direct GVM which modifies standard GVM's computational procedure to adapt to such alternative trends either increasing (Shu et al. 2011; Wang, Z.-X. et al. 2007; Wang, X.-Q. et al. 2014) or decreasing (Luo, He, Z.-M. 2009). Nevertheless, Wang, Y. et al. (2009) have managed to apply direct GVM to non-monotonic time series after complicated pre-processing procedure.

Table 1 summarises recent researches on GVM while its last few columns specify how this research is placed in the literature.

\section{Material and methods}

Subsection 2.1 explains research material, focusing on the population time series. We also take a glance at socio-economic time series, although their complete description is deferred to Supplementary information. Subsection 2.2 defines mathematical structure of traditional grey Verhulst model in standard form entitled GVM, its alternative formulation compatible with non-equidistant data identified as NGVM, and direct formulation of the latter model labelled DNGVM. The final Subsection 2.3 deals with proposed "Basic Form"-focused models. Accordingly, BFGVM, NBFGVM, and DNBFGVM are introduced, which stand for the basic proposed model, its non-equidistant alternative, and the direct version of the latter respectively. NBFGVMR and DNBFGVMR, improved versions of NBFGVM and DNBFGVM, are also presented. 


\subsection{Research material}

National censuses are the most reliable source for demographic research in Iran. Starting in 1956, they were administered every ten years, albeit intervals were halved from 2006 on; accordingly, a seven-point non-equidistant time series is available for Iranian population as provided in Table 2 .

Table 2. Iranian population data, in millions (Statistical Centre of Iran 2013)

\begin{tabular}{cccccccc}
\hline Year & 1956 & 1966 & 1976 & 1986 & 1996 & 2006 & 2011 \\
\hline Population & 18.954704 & 25.788722 & 33.708744 & 49.445010 & 60.055488 & 70.495782 & 75.149669 \\
\hline
\end{tabular}

Furthermore, our performance analysis also covers numerous domestic socio-economic time series. We include the most essential time series, so sub-national data - e.g. sectional (urban/rural), sectoral (primary/secondary/tertiary), and decile time series - as well as outdated data - i.e. the ones containing no information from 2006 on - are excluded. Moreover, time series that lead to inaccurate predictions from three or more models are pinpointed as inappropriate. Similar to Tsai et al. (2017), Lewis's threshold, i.e. a percentage error greater than 50 is adopted to detect inaccuracy. Lastly, time series with full linear dependence on others are also omitted to ensure each time series has enough statistical value. Final 19 time series are reported in Supplementary information in full detail.

\subsection{Formulating traditional models}

Overall procedure of formulating traditional GVMs is presented in Figure 1, while each formulation is described in a separate subsection.

\subsubsection{Formulating traditional GVM}

A defining characteristic of grey models is utilising sequence operations to reduce noise and facilitate information extraction (Hsin, Chen 2016; Wang, X.-Q. et al. 2014). $1^{\text {st }}$ order Accumulated Generation Operation (1-AGO) establishes $1^{\text {st }}$-order accumulated sequence $X^{(1)}$ by a simple summation of original sequence $X^{(0)}$. Fst-order Inverse AGO (1-IAGO), on the contrary, subtracts two successive values of $X^{(1)}$ to give $X^{(0)}$ (Wang, Y. et al. 2009). Finally, mean generation is an averaging operation on two successive sequence values:

$$
Z^{(1)}(t)=\frac{X^{(1)}(t)+X^{(1)}(t-1)}{2}=\frac{X^{(0)}(t)+2 X^{(1)}(t-1)}{2} .
$$

Each grey model is based on a differential equation named whitenisation equation. GM $(M, N)$ stands for standard grey model with a whitenisation equation of order $M$ involving 1 dependent plus $N-1$ independent variables (Liu, Lin 2010).

Forecasting procedure comprises a bipartite solution of whitenisation equation. First, its unknown parameters should be estimated. Least-squares computations necessitate approximating continuous whitenisation equation into discrete basic form equation. Then, 
finalised whitenisation equation is utilised at prediction. Its solution, i.e. time response function provides accumulated forecasts. Obviously, it should be processed by 1-IAGO to create restored values, the function providing non-accumulated forecasts.

Grey models usually rely on training dataset for estimating parameters, while a portion of data at the end called test dataset is excluded from training for validation. After getting trained by such data splitting, the grey model provides in-sample and out-ofsample results termed simulation and prediction respectively (Xiong et al. 2010; Zhou, He, J.-M. 2013).

However, GM $(M, N)$ is not all-inclusive; there are nonstandard grey models as well. Among such models, nonlinear grey Bernoulli models are especially popular. Assuming a power of two, GVM may be considered the basic nonlinear grey Bernoulli model.

Whitenisation equation and basic form of GVM are presented in Eqs. (2) and (3) respectively:

$$
\begin{aligned}
& \frac{d X^{(1)}(t)}{d t}+a X^{(1)}(t)=b\left(X^{(1)}(t)\right)^{2} ; t=1,2, \ldots, n, \\
& X^{(0)}(t)+a Z^{(1)}(t)=b\left(Z^{(1)}(t)\right)^{2} ; t=2,3, \ldots, n .
\end{aligned}
$$

Additional parameter estimation and prediction formulae may be found in Kayacan et al. (2010).

\subsubsection{Formulating traditional NGVM}

Herein, time itself contains non-trivial information. Let $t_{j}$ and $\Delta t_{j}$ denote the time sequence and its 1-IAGO. $\Delta t_{j}$ is composed of non-constant values as opposed to classical equidistant models with regular intervals of unity. More importantly, 1-AGO and 1-IAGO are revised to incorporate $\Delta t_{j}$ as demonstrated in Wang, Y.-H. et al. (2011).

\subsubsection{Formulating traditional DNGVM}

Direct NGVM shares its main structure with standard NGVM, but it differs in AGO and IAGO. DNGVM takes original input data as $X^{(1)}\left(t_{k}\right)$ and then applies 1-IAGO to it to make up $X^{(0)}\left(t_{k}\right)$. Likewise, DNGVM's time response function derives final forecasts directly, i.e. with no need for IAGO (Luo, He, Z.-M. 2009).

\subsection{Formulating proposed models}

Figure 1 depicts computational procedure shared between all proposed BFGVMs. It also distinguishes between traditional and proposed classes of models. This is complemented by individual descriptions of proposed models in following subsections.

Both classes discretise whitenisation equation to establish basic form equation, the basis of parameter estimation. Differences emerge when utilising estimated parameters. Traditional GVMs insert parameters back into continuous whitenisation equation and take its solution as the forecasting function, whereas proposed BFGVMs insert them into basic form itself, avoiding unnecessary skips between continuous and discrete computations. 


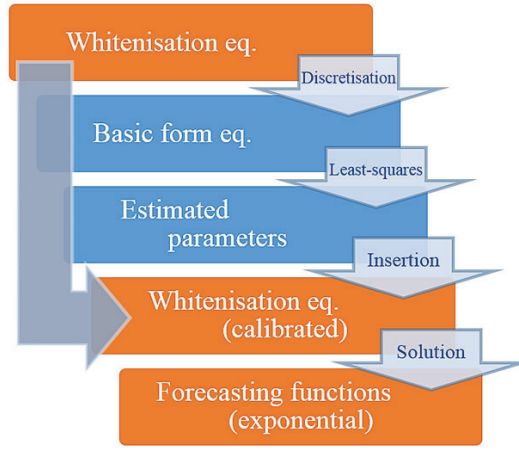

Traditional GVMs
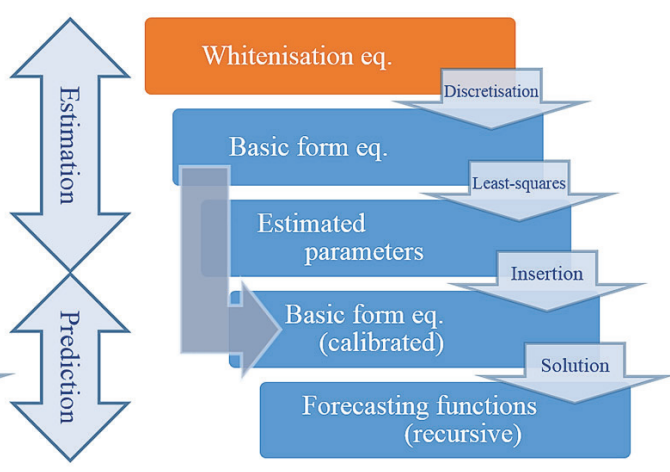

Proposed "Basic Form"-focused GVMs

Fig. 1. General computational procedure of traditional GVMs versus proposed BFGVMs

Note: orange and blue boxes denote continuous and discrete computations respectively.

\subsubsection{Formulating proposed BFGVM}

"Basic Form"-focused GVM resolves incompatibility between estimation and prediction processes of traditional GVM by taking discrete basic form equation as the common basis of both processes.

Using Eq. (1) to expand $Z^{(1)}(t)$ in GVM's basic form Eq. (3), we have

$-\frac{b}{4}\left(X^{(0)}(t)\right)^{2}+\left(1+\frac{a}{2}-b X^{(1)}(t-1)\right) X^{(0)}(t)+\left(-b\left(X^{(1)}(t-1)\right)^{2}+a X^{(1)}(t-1)\right)=0$.

Nonlinear right side of GVM's whitenisation Eq. (2) is the model's distinguishing characteristic, yet it imposes complications unfolding particularly herein; BFGVM's secondorder underlying Eq. (4) yields dual solutions, i.e. dual restored values:

$$
\begin{aligned}
& \hat{X}_{1}^{(0)}(t)=\frac{-2 b X^{(1)}(t-1)+(a+2)+\sqrt{(a+2)^{2}-8 b X^{(1)}(t-1)}}{b}, \\
& \hat{X}_{2}{ }^{(0)}(t)=\frac{-2 b X^{(1)}(t-1)+(a+2)-\sqrt{(a+2)^{2}-8 b X^{(1)}(t-1)}}{b} .
\end{aligned}
$$

Final restored values may not be determined unless such duality is resolved. We can compute outputs of both functions at all points. Negativity of a single output for a function implies that the other (all-positive) one is appropriate. In case non-negativity does not apply, we will consider accuracy measures to identify the appropriate function.

\subsubsection{Formulating proposed NBFGVM}

Being a non-equidistant model, unsurprisingly, NBFGVM involves forming the nontrivial sequence $\Delta t_{j}$. Furthermore, 1-AGO is revised in the same manner of traditional NGVM's. Assuming computational procedure of Subsection 2.3.1 to be approximately 
valid in non-equidistant settings, we make use of BFGVM's restored values with minimal modifications as follows:

$$
\begin{aligned}
& \hat{X}_{1}^{(0)}\left(t_{k}\right)=\frac{-2 b X^{(1)}\left(t_{k-1}\right)+(a+2)+\sqrt{(a+2)^{2}-8 b X^{(1)}\left(t_{k-1}\right)}}{b}, \\
& \hat{X}_{2}{ }^{(0)}\left(t_{k}\right)=\frac{-2 b X^{(1)}\left(t_{k-1}\right)+(a+2)-\sqrt{(a+2)^{2}-8 b X^{(1)}\left(t_{k-1}\right)}}{b} .
\end{aligned}
$$

\subsubsection{Formulating proposed NBFGVMR}

NBFGVMR is established by equipping NBFGVM with recursive simulation. It comprises employing time response function $\hat{X}_{1}^{(1)}\left(t_{k-1}\right)$ or $\hat{X}_{2}^{(1)}\left(t_{k-1}\right)$ instead of original accumulated time series $X^{(1)}\left(t_{k-1}\right)$ to compute subsequent simulated values, as

$$
\begin{aligned}
& \hat{X}_{1}^{(0)}\left(t_{k}\right)=\frac{-2 b \hat{X}_{1}^{(1)}\left(t_{k-1}\right)+(a+2)+\sqrt{(a+2)^{2}-8 b \hat{X}_{1}^{(1)}\left(t_{k-1}\right)}}{b}, \\
& \hat{X}_{2}{ }^{(0)}\left(t_{k}\right)=\frac{-2 b \hat{X}_{2}^{(1)}\left(t_{k-1}\right)+(a+2)-\sqrt{(a+2)^{2}-8 b \hat{X}_{2}^{(1)}\left(t_{k-1}\right)}}{b} .
\end{aligned}
$$

At prediction, differences between NBFGVMR and NBFGVM disappear since original data are non-available.

\subsubsection{Formulating proposed DNBFGVM}

Direct NBFGVM inherits standard NBFGVM's overall framework, although it makes two distinctions. First, same as DNGVM, original input data are taken as $X^{(1)}\left(t_{k}\right)$, while $X^{(0)}\left(t_{k}\right)$ is derived through 1-IAGO. Second, restored values are accumulated into time responses to derive final forecasts since the original time series was considered $X^{(1)}$ $\left(t_{k}\right)$ beforehand. Hence, we apply 1-AGO to NBFGVM's restored values to establish DNBFGVM's forecasting functions

$$
\begin{aligned}
& \hat{X}_{1}^{(1)}\left(t_{k}\right)=\hat{X}_{1}^{(1)}\left(t_{k-1}\right)+\frac{-2 b X^{(1)}\left(t_{k-1}\right)+(a+2)+\sqrt{(a+2)^{2}-8 b X^{(1)}\left(t_{k-1}\right)}}{b} \Delta t_{k}, \\
& \hat{X}_{2}{ }^{(1)}\left(t_{k}\right)=\hat{X}_{2}{ }^{(1)}\left(t_{k-1}\right)+\frac{-2 b X^{(1)}\left(t_{k-1}\right)+(a+2)-\sqrt{(a+2)^{2}-8 b X^{(1)}\left(t_{k-1}\right)}}{b} \Delta t_{k} .
\end{aligned}
$$

\subsubsection{Formulating proposed DNBFGVMR}

DNBFGVMR's computational procedure is identical to DNBFGVM's, while recursive simulation is the distinction. Similar to Subsection $2.3 .3, X^{(1)}\left(t_{k-1}\right)$ is replaced by $\hat{X}_{1}^{(1)}\left(t_{k-1}\right)$ or $\hat{X}_{2}^{(1)}\left(t_{k-1}\right)$ at simulation, whereas prediction remains intact.

$$
\begin{aligned}
& \hat{X}_{1}^{(1)}\left(t_{k}\right)=\hat{X}_{1}^{(1)}\left(t_{k-1}\right)+\frac{-2 b \hat{X}_{1}^{(1)}\left(t_{k-1}\right)+(a+2)+\sqrt{(a+2)^{2}-8 b \hat{X}_{1}^{(1)}\left(t_{k-1}\right)}}{b} \Delta t_{k}, \\
& \hat{X}_{2}^{(1)}\left(t_{k}\right)=\hat{X}_{2}^{(1)}\left(t_{k-1}\right)+\frac{-2 b \hat{X}_{2}^{(1)}\left(t_{k-1}\right)+(a+2)-\sqrt{(a+2)^{2}-8 b \hat{X}_{2}^{(1)}\left(t_{k-1}\right)}}{b} \Delta t_{k} .
\end{aligned}
$$




\section{Results}

This section compares traditional and proposed models. Aiming at ill-structured forecasting problems, we disregard equidistant models, i.e. simple GVM and BFGVM.

Subsection 3.1 explains our performance evaluation framework, i.e. error measures as well as short-term and long-term validation techniques, while Subsection 3.2 comprises actual comparative analyses.

\subsection{Performance evaluation method}

Numerous socio-economic time series are input to forecasting models, while we concentrate on Iranian census data, as discussed in Subsection 2.1.

Data splitting is applied with $65 \%$ and $35 \%$ quotas set for training and test datasets respectively, yet quotas are modified in Time Series 1 and 2 (Supplementary information) to keep the required minimum of four training data.

Multiple time series are analysed through nonparametric statistical tests. Wilcoxon signed-ranks and Friedman tests are selected for paired and multiple comparisons respectively as Demšar (2006) recommends. Both tests are conducted as two-way analyses.

Mean Absolute Percentage Error (MAPE) is widely employed in grey system research to measure simulation accuracy (Guo et al. 2013; Hsin, Chen 2016; Wang, Y.-H. et al. 2011; Xiao, Qin, L.-F. 2012), prediction accuracy (Wang, Y. et al. 2009), or both (Kayacan et al. 2010; Wang, Z.-X. et al. 2009; Wang, X.-Q. et al. 2014). Additionally, scale independence makes it preferable for analysis of multiple time series (Bergmeir, Benítez 2012). Hence, both simulation and prediction performance are measured in terms of MAPE.

However, data splitting cannot validate beyond two upcoming periods due to limited case study data. Accordingly, UN projections are taken as the benchmark (Table 3); they are substituted for non-available original data to validate population forecasts at farther horizons.

Table 3. World Population Prospects' projections for Iranian population, in millions (UN Population Division 2013)

\begin{tabular}{ccccccccccc}
\hline Year & 2016 & 2021 & 2026 & 2031 & 2036 & 2041 & 2046 & 2051 & 2056 & 2061 \\
\hline Projection & 80.460 & 84.995 & 88.764 & 91.938 & 94.746 & 97.242 & 99.327 & 100.850 & 101.690 & 101.851 \\
\hline
\end{tabular}

Nonparametric tests eliminate the need for benchmarking; accordingly, short-term and long-term predictions are not differentiated therein.

\subsection{Evaluating the performance of forecasting models}

Herein, comparative analyses are reported. Subsection 3.2.1 shows the overall picture of socio-economic results while deferring much information to Supplementary information; conversely, Subsection 3.2.2 describes results of the case study in more detail. 


\subsubsection{Performance in socio-economic time series}

Performance of all six models in socio-economic time series is summarised in Table 4 and Table 5 as well as Figure 2. These are subsequently input to nonparametric tests.

Table 6 through Table 9 followed by Table 10 and Table 11 briefly present results of Wilcoxon signed-ranks and Friedman tests respectively. Noticeably, average ranks of Friedman test are exactly consistent with scores of Wilcoxon paired comparisons in both simulation (Table 6 and Table 10) and prediction (Table 8 and Table 11).

Concerning simulation performance, Friedman test strongly rejects null hypothesis of equal medians among six models as Friedman and Iman-Davenport statistics are 67.99 and 45.32 respectively. Accordingly, post-hoc tests are performed to investigate superiority of the best simulator, i.e. DNBFGVM over others. Such tests reveal DNBFGVM to be better than all standard models, i.e. NGVM, NBFGVM, and NBFGVMR at least at $\alpha=3 \mathrm{e}-6$ according to Finner's and $\alpha=2 \mathrm{e}-6$ according to Li's procedure. Moreover, it is proved superior to DNGVM at $\alpha=0.024$ (Finner) or $\alpha=0.021$ (Li), though its advantage over runner-up DNBFGVMR is not verified.

Table 4. Simulation MAPEs in socio-economic time series

\begin{tabular}{|c|c|c|c|c|c|c|}
\hline Time series & NGVM & NBFGVM & NBFGVMR & DNGVM & DNBFGVM & DNBFGVMR \\
\hline 1 & 9.47 & 9.24 & 9.05 & 93.33 & 0.31 & 0.56 \\
\hline 2 & 31.89 & 31.31 & 56.02 & 1.45 & 1.41 & 1.40 \\
\hline 3 & 24.02 & 31.16 & 37.46 & 79.48 & 10.38 & 52.33 \\
\hline 4 & 69.08 & 32.99 & 86.20 & 4.64 & 4.16 & 3.98 \\
\hline 5 & 34.91 & 18.48 & 28.46 & 17.24 & 12.10 & 14.24 \\
\hline 6 & 12.05 & 13.00 & 12.25 & 1.08 & 0.96 & 1.09 \\
\hline 7 & 15.78 & 15.01 & 15.58 & 1.88 & 1.14 & 1.77 \\
\hline 8 & 15.86 & 16.04 & 14.85 & 3.96 & 3.99 & 3.95 \\
\hline 9 & 15.36 & 15.49 & 15.23 & $2.13^{3}$ & 1.91 & $2.13^{2}$ \\
\hline 10 & 15.34 & 14.50 & 15.10 & $1.75^{2}$ & 1.59 & $1.75^{3}$ \\
\hline 11 & 14.87 & 13.88 & 14.66 & $0.98^{3}$ & 0.91 & $0.98^{2}$ \\
\hline 12 & 17.04 & 14.86 & 16.61 & $2.57^{2}$ & 2.41 & $2.57^{3}$ \\
\hline 13 & 17.13 & 14.95 & 16.69 & $2.40^{2}$ & 2.33 & $2.40^{3}$ \\
\hline 14 & 16.27 & 14.86 & 16.17 & $2.52^{2}$ & 2.33 & $2.52^{3}$ \\
\hline 15 & 16.39 & 14.94 & 16.24 & $2.35^{2}$ & 2.30 & $2.35^{3}$ \\
\hline 16 & 18.40 & 16.95 & 18.24 & 0.19 & 0.16 & 0.12 \\
\hline 17 & 42.22 & 16.13 & 41.27 & 5.76 & 6.02 & 5.86 \\
\hline 18 & 22.53 & 18.36 & 22.32 & $5.51^{2}$ & 5.29 & $5.51^{3}$ \\
\hline 19 & 48.39 & 24.03 & 48.20 & 6.59 & 6.52 & 6.58 \\
\hline Mean & 24.05 & 18.22 & 26.35 & 12.41 & 3.49 & 5.90 \\
\hline Median & 17.04 & 15.49 & 16.61 & 2.52 & 2.33 & 2.40 \\
\hline
\end{tabular}

Note: Superscript numbers indicate exact ranks to disambiguate apparent ties. 
a)

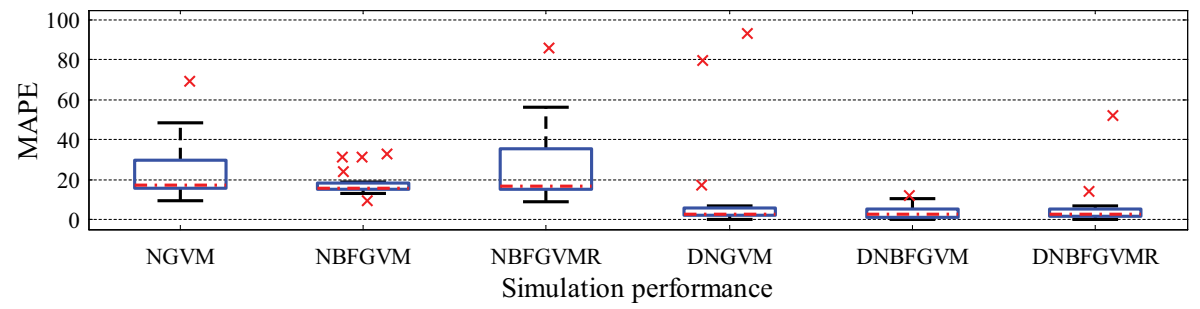

b)

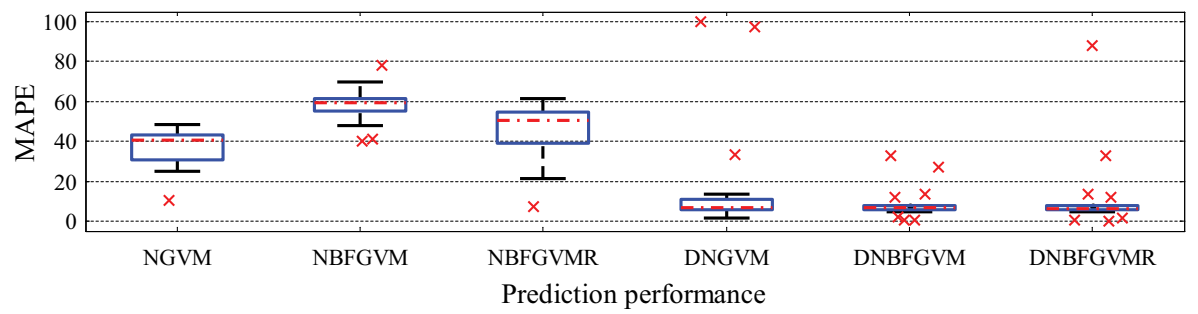

Fig. 2. Box plots of models' performance in socio-economic time series

Note: Parts (a) and (b) summarise in-sample and out-of-sample performance respectively.

Table 5. Prediction MAPEs in socio-economic time series

\begin{tabular}{|c|c|c|c|c|c|c|}
\hline Time series & NGVM & NBFGVM & NBFGVMR & DNGVM & DNBFGVM & DNBFGVMR \\
\hline 1 & 38.08 & 40.25 & 38.54 & 100.00 & 6.92 & 5.89 \\
\hline 2 & 45.51 & 78.17 & 7.26 & 1.60 & 0.29 & 0.14 \\
\hline 3 & 10.15 & 41.02 & 21.21 & 97.33 & 26.84 & 88.19 \\
\hline 4 & 25.43 & 48.12 & 55.64 & 3.32 & 0.60 & 0.59 \\
\hline 5 & 48.61 & 67.70 & 57.69 & 33.32 & 32.80 & 32.71 \\
\hline 6 & 47.06 & 62.51 & 59.50 & 1.69 & 1.92 & 1.67 \\
\hline 7 & 43.31 & 61.58 & 54.89 & 5.91 & 5.87 & 5.90 \\
\hline 8 & 39.21 & 55.00 & 48.68 & 5.87 & 5.94 & 5.90 \\
\hline 9 & 42.74 & 59.40 & 52.93 & 8.00 & 7.82 & 7.92 \\
\hline 10 & 41.23 & 59.91 & 53.96 & 5.56 & 6.03 & 5.55 \\
\hline 11 & 37.66 & 56.34 & 50.04 & 6.26 & 6.42 & 6.29 \\
\hline 12 & 40.48 & 59.15 & 50.33 & 6.08 & 6.61 & 6.06 \\
\hline 13 & 40.77 & 59.59 & 50.73 & 6.48 & 6.91 & 6.47 \\
\hline 14 & 41.26 & 60.28 & 51.61 & 6.67 & 7.29 & 6.65 \\
\hline 15 & 41.56 & 60.85 & 52.12 & 7.57 & 7.94 & 7.56 \\
\hline 16 & 46.56 & 69.81 & 61.40 & $6.69^{2}$ & $6.69^{3}$ & $6.69^{1}$ \\
\hline 17 & 24.96 & 53.30 & 33.25 & 11.93 & 11.88 & 11.89 \\
\hline 18 & 28.61 & 57.51 & 40.79 & $4.43^{2}$ & 4.71 & $4.43^{1}$ \\
\hline 19 & 25.52 & 60.94 & 32.27 & 13.56 & 13.53 & 13.55 \\
\hline Mean & 37.30 & 58.50 & 45.94 & 17.49 & 8.79 & 11.79 \\
\hline Median & 40.77 & 59.59 & 50.73 & 6.48 & 6.69 & 6.29 \\
\hline
\end{tabular}

Note: Superscript numbers indicate exact ranks to disambiguate apparent ties. 
Table 6. Scores of Wilcoxon test for simulation performance

\begin{tabular}{lcccccc}
\hline & NGVM & NBFGVM & NBFGVMR & DNGVM & DNBFGVM & DNBFGVMR \\
\hline NGVM & & 25 & 60 & 36 & 0 & 15 \\
\hline NBFGVM & 165 & & 175 & 37 & 0 & 17 \\
\hline NBFGVMR & 130 & 15 & & 35 & 0 & 13 \\
\hline DNGVM & 154 & 153 & 155 & & 16 & 54 \\
\hline DNBFGVM & 190 & 190 & 190 & 174 & & 163 \\
\hline DNBFGVMR & 175 & 173 & 177 & 136 & 27 & \\
\hline
\end{tabular}

Table 7. P values of Wilcoxon test for simulation performance

\begin{tabular}{|c|c|c|c|c|c|c|}
\hline & NGVM & NBFGVM & NBFGVMR & DNGVM & DNBFGVM & DNBFGVMR \\
\hline NGVM & & $\begin{array}{c}3.3 \mathrm{e}-3 \\
\star \star\end{array}$ & 0.169 & $\begin{array}{c}0.016 \\
\star\end{array}$ & $\begin{array}{l}3.8 \mathrm{e}-6 \\
\star \star\end{array}$ & $\begin{array}{l}5.2 \mathrm{e}-4 \\
\star \star \star\end{array}$ \\
\hline NBFGVM & $\begin{array}{c}3.3 \mathrm{e}-3 \\
\star \star\end{array}$ & & $\begin{array}{l}5.2 \mathrm{e}-4 \\
\star \star \star\end{array}$ & $\begin{array}{c}0.018 \\
\star\end{array}$ & $\begin{array}{l}3.8 \mathrm{e}-6 \\
\star \star\end{array}$ & $\begin{array}{l}7.9 \text { e-4 } \\
\star \star\end{array}$ \\
\hline NBFGVMR & 0.169 & $\begin{array}{l}5.2 \mathrm{e}-4 \\
\star \star \star\end{array}$ & & $\begin{array}{c}0.014 \\
\star\end{array}$ & $\begin{array}{l}3.8 \mathrm{e}-6 \\
\star \star\end{array}$ & $\begin{array}{l}3.4 \text { e-4 } \\
\star \star\end{array}$ \\
\hline DNGVM & $\begin{array}{c}0.016 \\
\star\end{array}$ & $\begin{array}{c}0.018 \\
\star\end{array}$ & $\begin{array}{c}0.014 \\
\star\end{array}$ & & $\begin{array}{l}6.4 \mathrm{e}-4 \\
\star \star \star\end{array}$ & 0.104 \\
\hline DNBFGVM & $\begin{array}{c}3.8 \mathrm{e}-6 \\
\star \star \star\end{array}$ & $\begin{array}{l}3.8 \mathrm{e}-6 \\
\star \star \star\end{array}$ & $\begin{array}{l}3.8 \mathrm{e}-6 \\
\star \star\end{array}$ & $\begin{array}{l}6.4 \mathrm{e}-4 \\
\star \star \star\end{array}$ & & $\begin{array}{c}4.6 \mathrm{e}-3 \\
\star \star\end{array}$ \\
\hline DNBFGVMR & $\begin{array}{l}5.2 \mathrm{e}-4 \\
\star \star \star\end{array}$ & $\begin{array}{l}7.9 \text { e-4 } \\
\star \star\end{array}$ & $\begin{array}{l}3.4 \text { e- } 4 \\
\star \star\end{array}$ & 0.104 & $\begin{array}{c}4.6 \text { e-3 } \\
\star \star\end{array}$ & \\
\hline
\end{tabular}

Note: Paired comparisons with significant differences at $0.001,0.01$, and 0.02 levels are indicated by three, two, and one star(s) respectively.

Table 8. Scores of Wilcoxon test for prediction performance

\begin{tabular}{lcccccc}
\hline & NGVM & NBFGVM & NBFGVMR & DNGVM & DNBFGVM & DNBFGVMR \\
\hline NGVM & & 190 & 171 & 37 & 4 & 19 \\
\hline NBFGVM & 0 & & 8 & 31 & 0 & 4 \\
\hline NBFGVMR & 19 & 182 & & 37 & 1 & 19 \\
\hline DNGVM & 153 & 159 & 153 & & 91 & 23 \\
\hline DNBFGVM & 186 & 190 & 189 & 99 & & 38 \\
\hline DNBFGVMR & 171 & 186 & 171 & 167 & 152 & \\
\hline
\end{tabular}


M. Hashem-Nazari et al. Non-equidistant "Basic Form"-focused Grey Verhulst Models (NBFGVMs) ...

Table 9. P values of Wilcoxon test for prediction performance

\begin{tabular}{|c|c|c|c|c|c|c|}
\hline & NGVM & NBFGVM & NBFGVMR & DNGVM & DNBFGVM & DNBFGVMR \\
\hline NGVM & & $\begin{array}{l}3.8 \mathrm{e}-6 \\
\star \star \star\end{array}$ & $\begin{array}{c}1.2 \mathrm{e}-3 \\
\star \star\end{array}$ & $\begin{array}{c}0.018 \\
\star\end{array}$ & $\begin{array}{l}2.7 \mathrm{e}-5 \\
\star \star\end{array}$ & $\begin{array}{c}1.2 \mathrm{e}-3 \\
\star \star\end{array}$ \\
\hline NBFGVM & $\begin{array}{l}3.8 \mathrm{e}-6 \\
\star \star \star\end{array}$ & & $\begin{array}{l}9.5 \mathrm{e}-5 \\
\star \star \star\end{array}$ & $\begin{array}{c}8.2 \mathrm{e}-3 \\
\star\end{array}$ & $\begin{array}{l}3.8 \mathrm{e}-6 \\
\star \star \star\end{array}$ & $\begin{array}{l}2.7 \mathrm{e}-5 \\
\star \star \star\end{array}$ \\
\hline NBFGVMR & $\begin{array}{c}1.2 \mathrm{e}-3 \\
\star\end{array}$ & $\begin{array}{l}9.5 \mathrm{e}-5 \\
\star \star \star\end{array}$ & & $\begin{array}{c}0.018 \\
\star\end{array}$ & $\begin{array}{l}7.6 \mathrm{e}-6 \\
\star \star \star\end{array}$ & $\begin{array}{c}1.2 \mathrm{e}-3 \\
\star \star\end{array}$ \\
\hline DNGVM & $\begin{array}{c}0.018 \\
\star\end{array}$ & $\begin{array}{c}8.2 \mathrm{e}-3 \\
\star\end{array}$ & $\begin{array}{c}0.018 \\
\star\end{array}$ & & 0.891 & $\begin{array}{c}2.4 \mathrm{e}-3 \\
\star\end{array}$ \\
\hline DNBFGVM & $\begin{array}{l}2.7 \mathrm{e}-5 \\
\star \star\end{array}$ & $\begin{array}{l}3.8 \mathrm{e}-6 \\
\star \star \star\end{array}$ & $\begin{array}{l}7.6 \mathrm{e}-6 \\
\star \star\end{array}$ & 0.891 & & $\begin{array}{c}0.020 \\
\star s\end{array}$ \\
\hline DNBFGVMR & $\begin{array}{c}1.2 \mathrm{e}-3 \\
\star\end{array}$ & $\begin{array}{l}2.7 \mathrm{e}-5 \\
\star \star \star\end{array}$ & $\begin{array}{c}1.2 \mathrm{e}-3 \\
\star\end{array}$ & $\begin{array}{c}2.4 \mathrm{e}-3 \\
\star \star\end{array}$ & $\begin{array}{c}0.020 \\
\text { है }\end{array}$ & \\
\hline
\end{tabular}

Note: Paired comparisons with significant differences at $0.001,0.01$, and 0.02 levels are indicated by three, two, and one solid star(s) respectively. Outline stars specify a 0.05 significance level.

Table 10. Sorted average rankings of Friedman test for simulation performance

\begin{tabular}{lc}
\hline Model & Ranking \\
\hline DNBFGVM & 1.3684 \\
\hline DNBFGVMR & 2.3158 \\
\hline DNGVM & 2.7895 \\
\hline NBFGVM & 4.2632 \\
\hline NBFGVMR & 4.8421 \\
\hline NGVM & 5.4211 \\
\hline
\end{tabular}

Table 11. Sorted average rankings of Friedman test for prediction performance

\begin{tabular}{lc}
\hline Model & Ranking \\
\hline DNBFGVMR & 1.5263 \\
\hline DNBFGVM & 2.3684 \\
\hline DNGVM & 2.6842 \\
\hline NGVM & 3.8421 \\
\hline NBFGVMR & 4.7895 \\
\hline NBFGVM & 5.7895 \\
\hline
\end{tabular}

Regarding prediction performance, Friedman test, once again, rejects the null hypothesis strongly as Friedman and Iman-Davenport statistics are 69.83 and 49.93 respectively. Post-hoc analyses confirm DNBFGVMR's prediction to surpass all standard models' at least at $\alpha=2.3 \mathrm{e}-4$ (Finner) or $\alpha=1.6 \mathrm{e}-4$ (Li). In addition, it is proved more accurate than DNGVM at $\alpha=0.070$ (Finner) or $\alpha=0.063$ (Li), even though it lacks meaningful advantage over runner-up DNBFGVM.

Furthermore, Wilcoxon test reveals each direct model to be superior over its standard alternative at simulation as well as prediction. It holds at $\alpha=0.02$ for traditional models and at $\alpha=0.002$ for proposed models.

It is hard to select between superior models, i.e. DNBFGVM and DNBFGVMR. Nevertheless, if we are to make a choice, it will be DNBFGVMR as prediction performance comes first.

Apparently, Friedman tests derive fewer significant results, but they are valuable verification of Wilcoxon tests as they control family-wise error of multiple pairwise comparisons (Demšar 2006). 


\subsubsection{Performance in the population case study}

Table 12 outlines results of the Iranian population case.

Between traditional NGVM and DNGVM, the latter is absolutely preferred.

Among proposed models, yet again, direct models are entirely preferable. Between direct proposed models, DNBFGVM excels at long-term prediction, while DNBFGVMR has simulation advantage.

Therefore, making a selection requires an eventual comparative analysis of traditional DNGVM as well as proposed DNBFGVM and DNBFGVMR.

As Table 12 and Figure 3 signify, proposed DNBFGVM and DNBFGVMR are far better population predictors with the former having edge in the long-term. Simulation results support superiority of these proposed models, albeit by smaller margins.

Table 12. MAPEs in forecasting Iranian population

\begin{tabular}{lcccc}
\hline Model & Class & Simulation & Short-term prediction & Long-term prediction \\
\hline NGVM & Traditional & 69.08 & 25.43 & 54.67 \\
\hline DNGVM & Traditional & 4.64 & 3.32 & 1.42 \\
\hline NBFGVM & Proposed & 32.99 & 48.12 & 95.86 \\
\hline NBFGVMR & Proposed & 86.20 & 55.64 & 61.15 \\
\hline DNBFGVM & Proposed & 4.16 & 0.60 & 0.90 \\
\hline DNBFGVMR & Proposed & 3.98 & 0.59 & 0.98 \\
\hline
\end{tabular}

a)

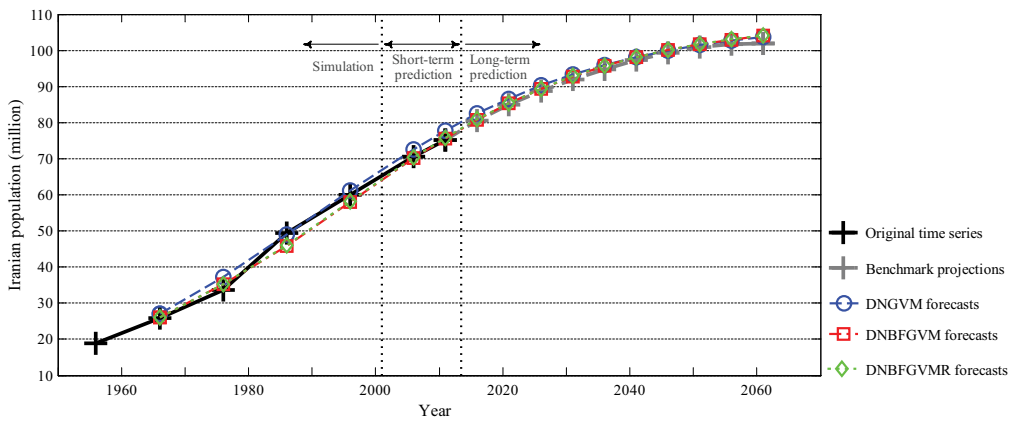

b)

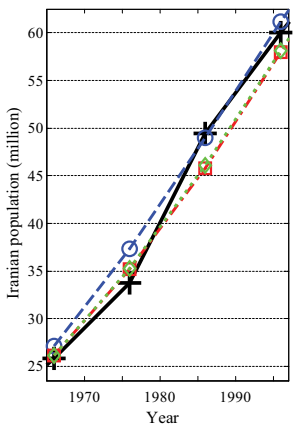

c)

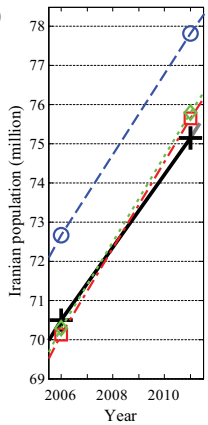

d)

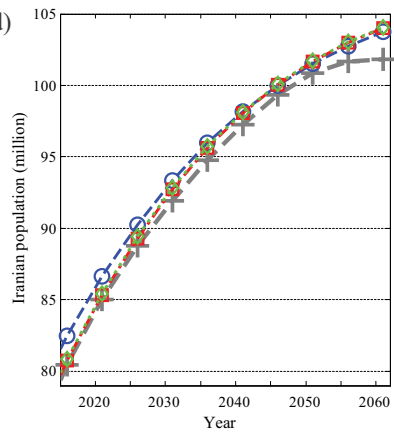

Fig. 3. Forecasts of Iranian population by direct models

Note: Part a) demonstrates forecasts as a whole, while parts b) through d) are its simulation, short-term prediction, and long-term prediction components respectively. 


\section{Discussion}

Results strongly support each direct model over its standard alternative, especially in the case study. Among direct models, traditional DNGVM is totally outperformed by proposed DNBFGVM and DNBFGVMR particularly in predicting the population. Socioeconomic evidence for significant excellence of two proposed models is even stronger as it covers not only prediction but also simulation.

Besides, nonparametric tests prove that recursive simulation typically improves prediction accuracy. Despite degrading simulation performance, it is recommended for standard and direct models alike.

To sum up, one can anticipate the best simulation from DNBFGVM and the most accurate prediction from DNBFGVMR. A comprehensive comparison may offer a selection in each case.

a)

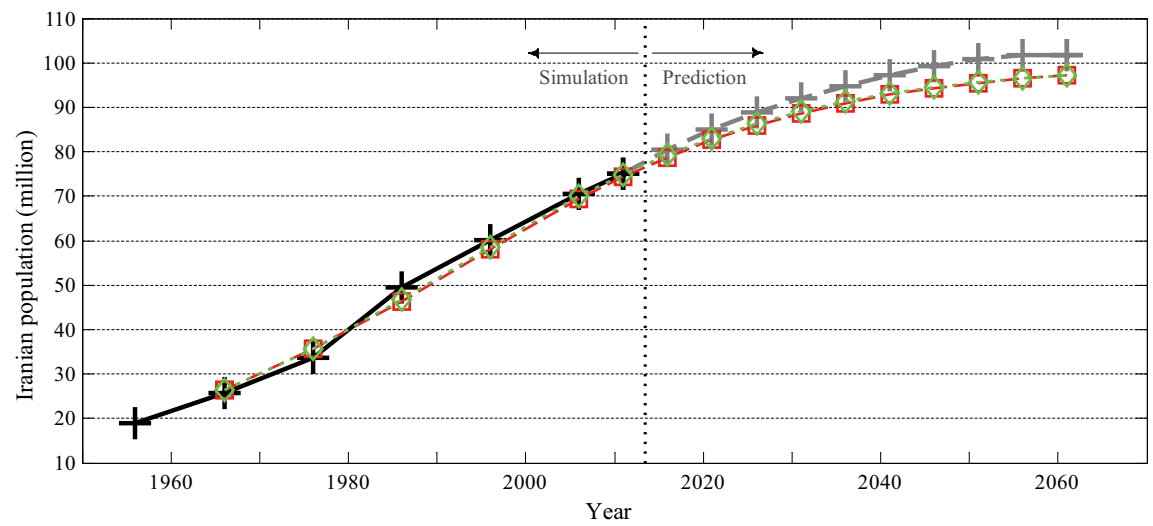

b)

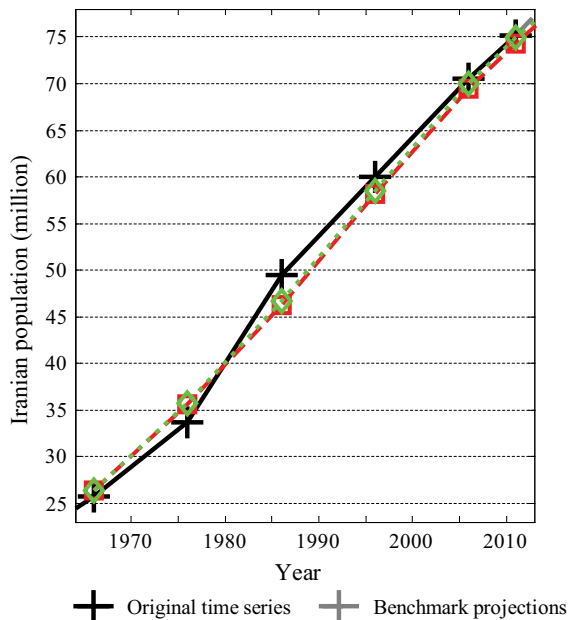

c)

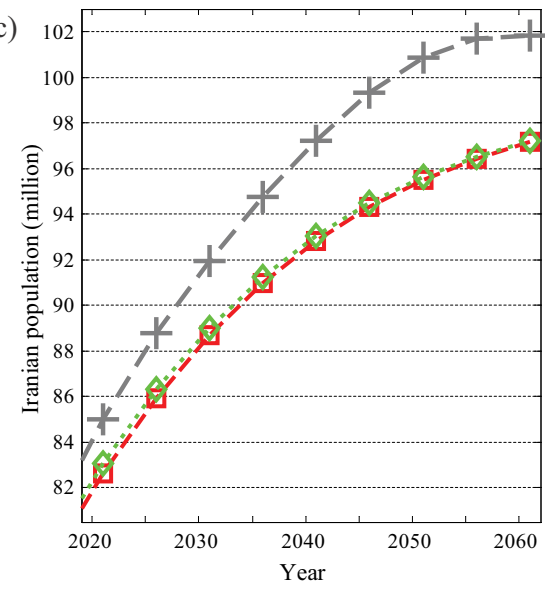

Fig. 4. Extrapolations of Iranian population by recommended models with all original data introduced into training

Note: Part a) draws the whole picture of forecasts, whereas parts b) and c) illustrate its simulation and prediction elements respectively. 
Having DNBFGVM and DNBFGVMR validated, we may remove data splitting to train them by all-data. The resulting couple of extrapolations establish upper and lower bounds of an interval forecast. For recently updated time series, they provide insights into upcoming years, yet for others, they estimate non-available recent and current data. Whereas Supplementary information details all extrapolations, Figure 4 depicts them for the case study.

Figure 4 depicts a gap between projections and forecasts which widens over time, while there is almost no such gap when data splitting is executed (Fig. 3). Whether projections have not taken full advantage of recent data or the gap has been formed otherwise should be investigated.

\section{Conclusions and recommendations}

Inherently affected by multiple uncertainties, socio-economic forecasting problems are not typically well-defined, especially when relying on poor data.

Herein, grey prediction was focused mainly due to its robustness to limited historical data. More specifically, Grey Verhulst Model (GVM) was concerned because it is adaptable to specific nonlinear trends.

The main contribution was to develop "Basic Form"-focused GVM (BFGVM), cohesive discrete forecasting procedure coordinating GVM's estimation and prediction. As far as we know, it was the first to bridge that gap while keeping GVM's configuration. BFGVM itself was then incrementally improved to suit a variety of ill-structured problems. It was adapted firstly to irregular time structure and secondly to violation of standard GVM's assumptions to establish Non-equidistant BFGVM (NBFGVM) and then Direct NBFGVM (DNBFGVM) respectively. Moreover, NBFGVM and DNBFGVM were equipped with "Recursive" simulation to develop NBFGVMR and DNBFGVMR respectively.

Models were applied to 19 socio-economic time series and examined by Wilcoxon signed-ranks and Friedman tests. The case of Iranian population was underlined due to its great strategic significance as well as its compatibility with parent Verhulst model setting a particularly appropriate foundation for testing GVM formulations.

Results were in favour of applying recursive simulation to either standard or direct models since prediction performance comes first.

More importantly, each direct model showed clear superiority over its standard alternative especially in the population case. It was true for both traditional - Non-equidistant GVM (NGVM) vs Direct NGVM (DNGVM) - and proposed - NBFGVM vs DNBFGVM or NBFGVMR vs DNBFGVMR - classes of models.

Consequently, DNGVM, DNBFGVM, and DNBFGVMR were qualified for the eventual comparative analysis. Population results signified that DNBFGVM and DNBFGVMR were far better than DNGVM in short-term and long-term prediction. More importantly, nonparametric tests revealed that even DNGVM's simulation was significantly outperformed by DNBFGVM. As a result, traditional DNGVM becomes obsolete by proposed 
DNBFGVM and DNBFGVMR, models recommended for socio-economic forecasting and particularly population forecasting.

Results imply that developed forecasting models can adapt to a variety of socio-economic time series with considerable accuracy even when they comprise ill-conditioned data. Thus, these models are invaluable tools for decision-makers especially when they cannot afford to appoint experts.

Extrapolations reveal that the population grows much slower than UN projects. UN projects, implying that implies that demographic consequences of the current family planning programme in Iran may be faced quite soon. These as well as other socioeconomic extrapolations provide precious practical implications.

This research has its limitations. Not every ill-structured socio-economic forecasting problem can be addressed by proposed models as it should roughly conform with GVM's structure. Additionally, the research was focused on specific gaps in GVM research, so the other gaps remain open. Finally, resolving the duality of forecasting functions for each proposed model incurs extra computational costs.

Future research is suggested to incorporate independent variables or parametric mean generation formulae into developed models, or to hybridise them with other forecasting techniques. Extending our core idea to nonlinear grey Bernoulli models is also promising. Developing systematic selection procedure between dual forecasting functions may realise much higher computational efficiency. Eventually, exploring proposed models may disclose other fields of their application.

\section{Acknowledgement}

We appreciate Mr. Yadollah Hashem-Nazari for his generous help to improve English of this paper.

\section{References}

Bergmeir, C.; Benítez, J. M. 2012. On the use of cross-validation for time series predictor evaluation, Information Sciences 191: 192-213. https://doi.org/10.1016/j.ins.2011.12.028

Bezuglov, A.; Comert, G. 2016. Short-term freeway traffic parameter prediction: application of grey system theory models, Expert Systems with Applications 62: 284-292.

https://doi.org/10.1016/j.eswa.2016.06.032

Demšar, J. 2006. Statistical comparisons of classifiers over multiple data sets, Journal of Machine Learning Research 7: 1-30.

Erfani, A. 2013. A reversal in the population policy of Iran: do curbing family planning programs raise low fertility?, in XXVII IUSSP International Population Conference, 27 August 2013, Busan, Republic of Korea.

Esfahanipour, A.; Goodarzi, M.; Jahanbin, R. 2016. Analysis and forecasting of IPO underpricing, Neural Computing and Applications 27(3): 651-658. https://doi.org/10.1007/s00521-015-1884-1

Evans, M. 2014. An alternative approach to estimating the parameters of a generalised Grey Verhulst model: an application to steel intensity of use in the UK, Expert Systems with Applications 41(4, Part 1): 1236-1244. https://doi.org/10.1016/j.eswa.2013.08.006

Guo, X.-J.; Liu, S.-F.; Fang, Z.-G. 2013. Study on a grey Verhulst self-memory model and application, in IEEE International Conference on Grey Systems and Intelligent Services (GSIS), 15-17 November 2013, Macao, 118-122. 
Hsin, P.-H.; Chen, C.-I. 2016. Application of trembling-hand perfect equilibrium to Nash nonlinear Grey Bernoulli model: an example of BRIC's GDP forecasting, Neural Computing and Applications (in press). https://doi.org/10.1007/s00521-016-2340-6

Hu, B.; Qin, Y.-L. 2011. Forecast on Shanghai's input-intensity of R\&D based on the model DGM $(1,1)$, in International Conference on Management and Service Science (MASS), 12-14 August 2011, Wuhan, China, 1-4.

Jafari, H.; Pourreza, A.; Vedadhir, A.; Jaafaripooyan, E. 2017. Application of the multiple streams model in analysing the new population policies agenda-setting in Iran, Quality \& Quantity 51(1): 399-412. https://doi.org/10.1007/s11135-016-0311-8

Kayacan, E.; Kaynak, O. 2011. Single-step ahead prediction based on the principle of concatenation using grey predictors, Expert Systems with Applications 38(8): 9499-9505.

https://doi.org/10.1016/j.eswa.2011.01.143

Kayacan, E.; Ulutas, B.; Kaynak, O. 2010. Grey system theory-based models in time series prediction, Expert Systems with Applications 37(2): 1784-1789.

https://doi.org/10.1016/j.eswa.2009.07.064

Li, G.-D.; Wang, C.-H.; Masuda, S.; Nagai, M. 2011. A research on short term load forecasting problem applying improved grey dynamic model, International Journal of Electrical Power \& Energy Systems 33(4): 809-816. https://doi.org/10.1016/j.ijepes.2010.11.005

Liu, S.-F.; Lin, Y. 2010. Grey systems: theory and applications. Berlin: Springer. https://doi.org/10.1007/978-3-642-13938-3

Luo, Y.-X.; He, Z.-M. 2009. The non-equal-interval direct new information Verhulst GM(1,1) model with two times fitting and its application to test data processing, in 2nd International Conference on Power Electronics and Intelligent Transportation System (PEITS), 19-20 December 2009, Shenzhen, China, 85-88. https://doi.org/10.1109/PEITS.2009.5406958

Mahmoodi, K.; Mohammadpur, A.; Rezaei, M. 2015. A discourse analysis of population policies in the context of politics in Iran, Quality \& Quantity 49(5): 1883-1895.

https://doi.org/10.1007/s11135-014-0080-1

Shu, H.; Dang, Y.-G.; Xiong, P.-P. 2011. Research on characteristics of grey non-equidistant Verhulst model, in IEEE International Conference on Grey Systems and Intelligent Services (GSIS), 15-18 September 2011, Nanjing, China, 209-212. https://doi.org/10.1109/gsis.2011.6044145

Statistical Centre of Iran. 2013. Country population, [online], [cited 22 January 2017]. Available from Internet: https://www.amar.org.ir/Portals/1/PropertyAgent/2212/Files/2575/country\%20 population.xlsx

Tsai, S.-B.; Xue, Y.-Z.; Zhang, J.-Y.; Chen, Q.; Liu, Y.-B.; Zhou, J.; Dong, W.-W. 2017. Models for forecasting growth trends in renewable energy, Renewable and Sustainable Energy Reviews 77: 1169-1178. https://doi.org/10.1016/j.rser.2016.06.001

UN Population Division. 2013. World population prospects: the 2012 revision. A report commissioned by the Population Division of the Department of Economic and Social Affairs.

Vahidnia, F. 2007. Case study: fertility decline in Iran, Population and Environment 28(4-5): 259-266. https://doi.org/10.1007/s11111-007-0050-9

Wang, X.-Q.; Qi, L.; Chen, C.; Tang, J.-F.; Jiang, M. 2014. Grey system theory based prediction for topic trend on internet, Engineering Applications of Artificial Intelligence 29: 191-200. https://doi.org/10.1016/j.engappai.2013.12.005

Wang, Y.-H.; Dang, Y.-G.; Pu, X.-J. 2011. Improved unequal interval grey model and its applications, Journal of Systems Engineering and Electronics 22(3): 445-451.

https://doi.org/10.3969/j.issn.1004-4132.2011.03.012 
Wang, Y.; Song, Q.-B.; Macdonell, S.; Shepperd, M.; Shen, J.-Y. 2009. Integrate the GM(1,1) and Verhulst models to predict software stage effort, IEEE Transactions on Systems, Man, and Cybernetics - Part C: Applications and Reviews 39(6): 647-658. https://doi.org/10.1109/TSMCC.2009.2020690

Wang, Z.-X.; Dang, Y.-G.; Liu, S.-F. 2009. Unbiased grey Verhulst model and its application, Systems Engineering - Theory \& Practice 29(10): 138-144.

https://doi.org/10.1016/S1874-8651(10)60078-6

Wang, Z.-X.; Dang, Y.-G.; Wang, Y.-M. 2007. A new grey Verhulst model and its application, in IEEE International Conference on Grey Systems and Intelligent Services (GSIS), 18-20 November 2007, Nanjing, China, 571-574.

Xiao, X.-P.; Qin, L.-F. 2012. A new type solution and bifurcation of grey Verhulst model, Journal of Grey System 24(2): 165-174.

Xie, N.-M.; Liu, S.-F. 2009. Discrete grey forecasting model and its optimization, Applied Mathematical Modeling 33(2): 1173-1186. https://doi.org/10.1016/j.apm.2008.01.011

Xiong, P.-P.; Dang, Y.-G.; Qian, W.-Y. 2010. The optimization of time response function in nonequidistant Verhulst model, Journal of Grey System 22(3): 249-256.

Zhou, W.; He, J.-M. 2013. Generalized GM $(1,1)$ model and its application in forecasting of fuel production, Applied Mathematical Modeling 37(9): 6234-6243.

https://doi.org/10.1016/j.apm.2013.01.002

Mohammad HASHEM-NAZARI is a graduate of prestigious Allameh Helli High School. He received B.Sc. and M.Sc. in industrial engineering from K.N.Toosi University and Tarbiat Modares University respectively. Now, he is a $\mathrm{PhD}$ candidate in industrial engineering at Amirkabir University of Technology. His research interests include grey prediction and customer relationship management.

Akbar ESFAHANIPOUR studied industrial engineering to receive B.Sc. from Amirkabir University of Technology and M.Sc. and PhD from Tarbiat Modares University, followed by a Postdoctoral Fellowship at DeGroote School of Business, McMaster University. He is now an Assistant Professor of industrial engineering at Amirkabir University of Technology with research interests in financial time series forecasting, decision-making, and risk analysis.

S.M.T. FATEMI GHOMI received B.Sc. and $\mathrm{PhD}$ in industrial engineering from Sharif University of Technology and University of Bradford respectively. Now, he is a Professor of industrial engineering at Amirkabir University of technology. His interests involve time series analysis, stochastic activity networks, production planning and control, scheduling, queueing systems, and statistical quality control. 